\title{
A Critical Insight into Congestion Control Mechanism in WSN
}

\author{
Vikas Srivastava ${ }^{1}$, Karan Singh ${ }^{2}$ and Sachin Tripathi ${ }^{3}$ \\ ${ }^{1}$ Dept. of CSE, IIT (ISM) Dhanbad \\ ${ }^{2}$ School of Computer \& System Science, JNU New Delhi \\ ${ }^{3}$ Dept. of CSE, IIT (ISM) Dhanbad \\ ${ }^{1}$ sriv.vikas@gmail.com, ${ }^{2}$ karan@mail.jnu.ac.in, ${ }^{3}$ var_1285@yahoo.com
}

\begin{abstract}
The wireless network leads towards the unpredictable network load due to the eventdriven nature of WSN. The sensors may densely distribute in different areas i.e. in the Forest, in militaries and etc. The sensor nodes carry all necessary components for transmission and reception of data packets. During the transmission, the data packet may be lost due to the congestion at single node. In this paper, we review, classify and compare the approaches of various congestion control mechanisms through which the issues have been fairly controlled based on assumptions and results provided by various researchers.
\end{abstract}

Keywords: Base Station (BS); Wireless Sensor Networks; Congestion; Routing

\section{Introduction}

One or more sinks and thousands of sensor nodes which are scattered, form a Wireless Sensor Network (WSN) where sensors sense and process the information for the sinks. Sensors in WSN are very tiny with limited energy and computing resources and sense the different physical and environmental conditions such as temperature, pressure, motion and pollution at different locations. These days' applications are increasing in the area of earthquake, railways, defense, health, fire alarming, tracking and smart houses. Due to the rapid growth of sensor based applications, congestion becomes an utmost challenge in WSN which arises due to upstream traffic or downstream traffic. The network traffic from sensors nodes to sink is upstream and vice versa is downstream traffic. There are enormous research exits to justify the need to control congestion in WSNs. Initially lots of cases have been taken up in the area of physical layer, MAC layer [34], transport layer [32] and network layer. Afterwards, a lots of attention has been paying in challenges of congestion control mechanisms. Some of studies [12] asked about on this issue with support of numerical results while some of researchers [20, 22] have analyzed and provide specific solutions of the problem. The paper [21] emphasizes on congestion control techniques in some constrained environment by improving Constrained Application Protocol while $[9,11]$ reviews a number of congestion control protocols, algorithms and able to compare the congestion control algorithms, congestion avoidance algorithms and reliable data transport mechanisms. The future directions relevant to different applications were also been discussed with focus on performance driven and controlled mechanism.

This paper is a survey paper which does not claim any research contribution. A comprehensive survey has been conducted from the various reputed journals like IEEE, IEEE Commun Letter, IEEE Symposium, ACM, EURASIP, Journal of Communications and IEEE conferences proceedings in the span of last 5-6 years. The objective of paper is to provide a critical insight of considerable contribution in different approaches to control congestion, proposed for WSNs. This paper elaborates the characteristics as well as merits and demerits of different approaches. Various categorizations among the different types 
of approaches to control congestion are based on i) congestion detection ii) congestion avoidance iii) and congestion mitigation. Finally impact of congestion in WSNs on packet loss and energy loss discussed and try to provide specific directions on relevant issues to the forthcoming researchers for the planning and development of pioneering congestion control mechanisms.

The organization of paper is: First we understand and define the scenarios that motivates congestion control in Section II, how congestion occurs in WSN and its probable issues are discussed in Section III, the conventional approaches is discussed in Section IV, make survey on existing methods and limitations of existing approaches in Section V, discussion for this survey is presented in Section VI while Section VII and VIII presents an opinion of the future guidelines and final observations correspondingly.

\section{Motivation}

In current segment, we take up the scenarios with which we inspired of congestion manage in WSNs, and explain how consistency and data accessibility is improved. Describing the congestion, most commonly observation is data pursue a tree created routing topology with BS as a source of the tree presented in Figure 1. The parent node $S_{i}$ represents sink while the sibling nodes in Figure represent as generators / or forwarders as mentioned in previous section.

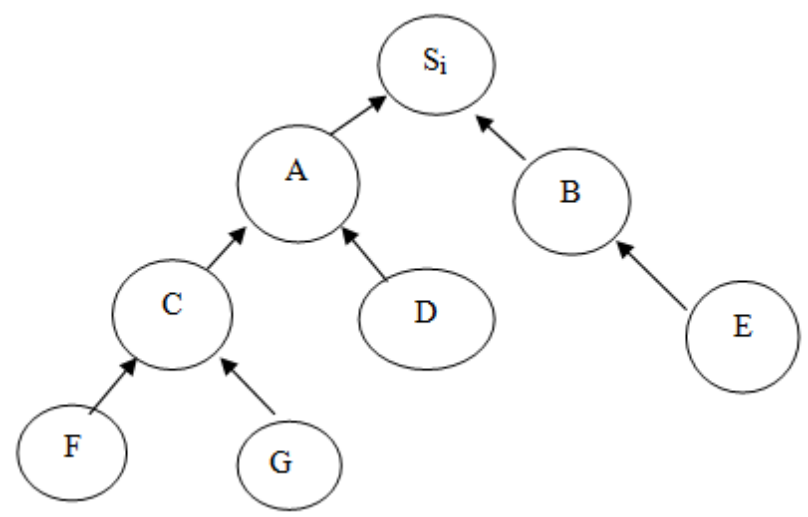

Figure 1. Tree Structure Based Routing Topology

The upstream nodes are close to the source nodes while downstream nodes are near the sink. As shown in Figure, nodes F and G represents upstream node for node C, whereas for node $\mathrm{C}$, node $\mathrm{A}$ termed to be as downstream node.

It has been understood, packet collision, channel conflict, interference, buffer overflow and many-to-one flow nature included as the main sources of congestion. Buffer overflow happens when the quantity of inward packet is larger than the existing buffer space. Contention takes place among diverse flows and unlike packets of a flow. Interference is originated through concurrent communications with several paths of the network among the nearby nodes [20,3]. Packet collisions lead to packet drops, hence lower level congestion. Congestion is a cause of quality degradation, packet losses and packet drops based on the applicable topology and position of nodes in WSNs. The effect of congestion represented as: - 


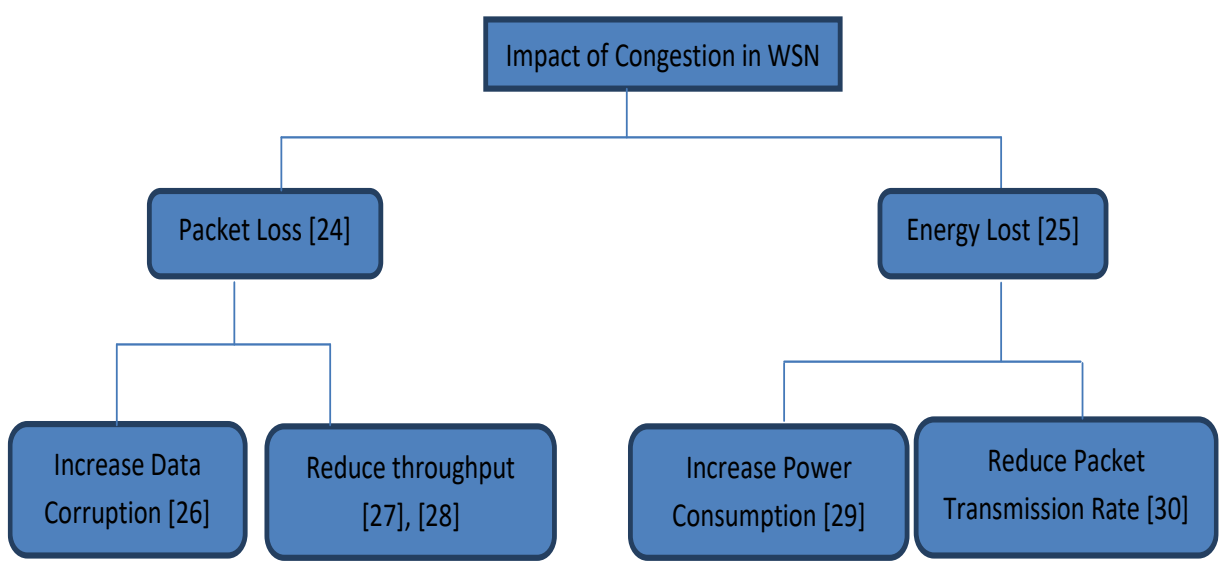

Figure 2. Congestion Impact in WSN

In congestion, the temporary load is bigger than the network resources handles in a way that resources turn out to be useless. The importance of packet loss consideration has been analyzed [19] within inter node communication in WSN through network utilization phase. To mitigate this, the best communication path is considered. The energy consumption is one of the constraint,[25] shows and contrast numerous Media access control and routing protocols that have been designed to optimize the power utilization without negotiating the data delivery in WSNs. To detect data anomalies with faulty nodes [7] proposes innovative technique FIND to recognize nodes with data errors that neither presume a particular sensing model nor require costly event injection. Under the proposal they have been able to achieve their target with 5\%. Reduced throughput is another challenge due to congestion, $[18,31]$ significantly able to improve the throughput performances by their proposed methods. The power utilization is the main concern for the development of a WSN solutions, [15] proposes solution in order of assessment of power utilization of wireless sensor networks by migrated to cloud computing environment. To maintain optimized data flow during transmission in WSN is also one of the challenges which [23] is able to achieve significantly by applying Principal Component Analysis (PCA) algorithm.

As per observations, in next section, we will provide more comprehensive study on the effect of congestion and extract more detailed conclusions of various approaches which have been researched for congestion.

\section{Congestion in WSNs}

Wireless sensor networks (WSNs) is a collection of "nodes" - a small embedded device which interfaces with sensors and do communication with small range wireless transmitters, from few to hundreds or thousands. The node is grouped in a rational way in which data packets are directed hop-by-hop towards sinks or BS. Based on the applications, WSNs generate large enough information which should be conveyed to a tiny amount of sinks. This rate of data may lead to congestion. Due to congestion, various issues and its impact is being observed, which are classified under the following categories: -

Packet Loss:- It occurs in the specified area of WSN, many nodes try to send the data concurrently which resulting the losses due to increase of data size near sync in network and sinking throughput of all nodes. The packet loss can be reduced by time synchronization between nodes. The packet losses nearby Source to source, sink to sink and may be due to lose energy. By nature, WSNs are mostly unprotected and medium is broad. The impact of decreasing energy increases the power consumption and reduced the packet rate influenced packet loss in WSN. 
Packet Drops: - In a particular area, buffer used to hold the data for further process. The buffer overflows with higher rate of data transmission.

Congestions have been classified as: -

i) Node-level Congestion

In traditional networks, node-level congestions is common in nature due to overflow of buffer in the node which results loss of packets and increases queuing delay.

ii) Link-level congestion:

In a particular area, the collision happen when several live source nodes are trying to transmit data on similar instance within range of one another. It may be notify that packets leave the buffer, are not succeeded to arrive at the next hop due to collision. It increase packet delay and energy waste in spite of decreasing link utilization and overall throughput [30].

\section{Approaches}

Congestion control is a technique utilize to observe the procedure of guiding full quantity of information inflowing to the network to maintain traffic intensity at an adequate value. It manages and control the traffic access into the system to keep away any congestive collapses of data by reducing data transfer rate to transmit data packets from Multiple node to the BS.

There are conventional methods to handle congestion: Network resource management and Traffic control. Network resource management attempts to extend network resource to mitigate congestion control, while during the congestion in network, mitigation algorithms must considered to take instant actions to control.

In a periodic traffic, generally behaviour of sensor nodes are static and traffic rate is low by nature. There may be extensive idle periods in which sensor nodes turn off their radio and set out to sleep to save energy consumed by idle listening [35]. It is required to implement algorithms to send the data over MAC layer and network layer to control the traffic at different levels on available bandwidths. There are some available common methods to control traffic in WSNs: Hop-by-hop congestion control and end-to-end control which highlights in literature reviews.

Various algorithms have taken up both approaches in their attempt to handle congestion. A hybrid method can be attempted to trade on the approaches.

\section{Related Works}

Due to the limited bandwidth of WSN, it faces congestion frequently during the transmission of sensing data. Congestion control has to consider network capacity and application requirements. The various schemes were proposed by researchers to address these challenges i.e. Local Cross Layer Congestion Control, Adaptive Duty Cycle based Congestion Control, Receiver Assisted Congestion Control, Congestion Avoidance and Fairness (CAF), Light Weight Buffer Management (LWBM) etc.

James Dzisi Gadze et al. proposed Adaptive congestion control protocol (ACCP) for WSN, which represents the adaptive congestion control algorithm to monitor network utilization and adjust traffic levels and/or increase network resources to improve throughput and conserve energy. Backpressure mechanism has been introduced into NO Ad-Hoc Routing Agent (NOAH) with the development of traffic congestion control logic (DelStatic). It has been established that DSR has a higher resource congestion control capability based on its Node Rank. In proposed ACCP, sink switched algorithm is used to trigger DelStatic. Simulation conducted in NS2 on various simulation parameters: Transmission range, Interference range, MAC protocol, max. packet in queue, energy parameters taken into an account, the results showed that ACCP not only able to achieved reliability, high throughput but also conserves energy which is critical to sensor application survivability on the field [14]. 
Chonggang Wang et al. proposed Priority Based Congestion Control (PCCP), a hopby-hop upstream congestion control protocol used the basic components: intelligent congestion detection (ICD), implicit congestion notification (ICN), and priority-based rate adjustment (PRA) to reduce packet loss to guarantee fairness in multipath routing. PCCP defines a variable congestion degree as a ratio of average packet service time over average packet inter arrival time on a node. It identifies the level of current congestion at each sensor node. ICN notify the congestion information in the header to be forwarded by taking advantage of broadcast nature of wireless channel. The PRA used to have the focus to get the higher priority index and equal bandwidth for sufficient traffic. With PCCP, a number of up streams data sources can be find out and able to measure the downstream forwarding rate. It helps to measure congestion accurately by priority index and thus able to achieve high link deployment and flexible fairness at tiny buffer size [33].

Muhammad Monowar et al. presented Prioritized Heterogeneous Traffic-oriented Congestion Control Protocol (PHTCCP), a proficient congestion control mechanism for heterogeneous data originated from multipurpose sensor nodes. A variable Packet service ratio r(i) has been used to compute the congestion level at each sensor node. By implicit congestion notification (ICN), it able to hear the congestion information because of broadcast nature of wireless channel. Rate adjustment is triggered to control the congestion and ensures efficient rate for the prioritized diverse traffic. Inter-intra queue priorities is used to ensure feasible transmission of heterogeneous data. The mechanism is simulated on NS2 simulator and achieved desired throughput, high link utilization and reduced packet loss by using dynamic transmission rate. The results also ensure its energy efficiency and lower delay [16].

In WSNs, TDMA and contention based access are two approaches. Contention based MAC protocols achieved low power operation by applying duty cycling mechanism. In another approach, node must synchronize its active periods with neighbouring node to awake at the same time. A large quantity of slots in a frame may lead to message delay in network due to which TDMA approach is inappropriate for densely-deployed WSNs and energy waste is high in heavy traffic load. Researchers able to demonstrate the collision free network through conflict-free slot assignment in traditional TDMA protocols where as reduced frame approach were analyzed on performance and energy consumption. The performance of reduce framework were increased by tuning the frame size and reduced the number of conflicting slot assignment via heuristic slot assignment algorithm. The proposal concluded to evaluate the scalability of network density and self organized adaptive reduced frame TDMA schemes as future directives [8].

In WSN, Fairness - Aware Congestion Control (FACC) Scheme by Xiaoyan Yin et al. proposed a rate-based congestion control protocol to control congestion and achieve fair bandwidth allocation for different flows. An intermediary sensor nodes has been categorized to near-source nodes and near-sink nodes.

Per-flow state is preserved to near source nodes and allocate fair rate to each passing flow by comparing the incoming rate of each flow and the fair bandwidth share. Near source nodes were govern based on following metrics: -

- Available Bandwidth evaluation.

- Flow Arrival Rate Computation.

- Number of live Flows Estimation.

- Near-Source Nodes - Transmission Control

On the other side, close to- BS node, there is no need to maintain state of each flow and apply probabilistic dropping algorithm based on hot frequency. It implemented basic method: A Stateless Fair Queue Management Mechanism, a Hop-by-Hop Backpressure mechanism and Fairness of the Stateless Queue-Management mechanism. To achieve fairness, it gives more chances to lower occupancy. Thus inward packets corresponds to superior occupancy flows, having greater sinking possibility by Hop-by-Hop 
backpressure mechanism. FACC notifies the near-source node to maintain each flow state and assign the fair rate to each flow [30].

To address issues of packet loss, throughput, waste of energy, Majid Gholipour et al. proposed distributed traffic-aware routing scheme with capacity of adjustment of data transmission rates for multi-sink wireless sensor networks from source to sink nodes is proposed. The algorithm constructed on parameters depth field and traffic loading, respectively. Depth field represents the length Depth (v) of shortest path from the distance. The author given proofing of loop free shortest path routing to encourage packet to flow straight to the sink. The second parameter define three basic factors: queue length, congestion degree, and average cumulative queue length to improve overall throughput. Once the traffic loading exceeds a threshold, it shows the congestion at a node in the path toward a specific sink; packets flow along to other optimal paths. The weighted factors applied on network to reduce computation resource at sensor nodes, improves network performance. The proposal concluded with simulation results indicating improved network performance, end-to-end packet delay, energy consumption and packet delivery ratio compared to other congestion control schemes [i.e. CODA, SPF, ESRT]. However it overhead the resources to calculate every flow for every time to get optimal result value for weighted factors [4].

Mohammed Ashraf. A et al. proposed Flow Deviation Congestion Control (FDCC) to control the congestion efficiently in the network via a control message in the node where packet is drop. The performance metric of scheme is based on three fields flow id, node id and destination id. The algorithm proceeds in identifying the routing flows available. It select the alternative path in case of flow found to be busy and node id where packet is dropped and also compare the node with neighbour node in free path to forward warning message with destination address until it reach to the sink node. The mathematical model of scheme shown improvement of network performance, data transfer, data loss by applying waiting model and gain speed of packet transmission from sensor nodes to source nodes [6].

Multipath Routing With Novel Packet Scheduling implement multipath routing protocol to enable the reliable data delivery in a sensor network environment. The scheme uses a prioritizer at network layer to place data in the appropriate queue. Each packet is assigned a priority number to schedule for transmission based on priority. An Earliest deadline first (EDF) algorithm is used to assign priorities and it also search the closest process which in turn to be schedule for execution. It uses the queuing model for a node of inter and intra queue priority. It helps in identifying the node with priority number and the packets header information. EDF algorithm, schedule the transmission of selected high priority packet. It also involve the traffic distribution, path discovery and path maintenance. The optimal solution of proposed scheme increases the packet service ratio and decreases packet drop. The performance evaluation of schemes in busty traffic is still unexplored. [26].

As delay-throughput performance in TCP and its poor capability to utilize the network bandwidth, Receiver Assisted Congestion Control Mechanism (RACC) has been proposed to resolve congestion issues. RACC allow the receiver to participate in congestion control for which a timer is implemented at the receiver to detect packet drop. The receiver send ACK earlier to inform the sender about the timeout which help to reduce the waiting time of the sender to retransmit a lost packet. The scheme measures the bandwidth, then computers an appropriate congestion window size based on the measured bandwidth. The researchers compare the mechanism with TCP NewReno and TCP Westwood at different scenarios i.e. network performance, wired delays on the throughput [35].

Due to limited buffer capacity of WSNs, the network throughput degraded in WSNs. Adaptive Buffering Scheme (ABS) proposed allocation and buffering of sensor-generated data packets at source nodes. However it also propose to source node, must store the data 
for some extended slots of time period to reduce network congestion. One of the parameter Forwarding packets with Mobile Sink (MS) is proposed in predetermined path to balance the traffic load. It identify the neighbour nodes to buffer the data packet and source node will continue to forward data packet till chosen path becomes full. Both the concepts has been proven by Mathematical model. In support to resolve these issues The simulation tool ("OMNeT++") is used to compare the performance as well as it proves effective performance in reducing the packet loss and average end-to-end delay [17].

Table 1. Congestion Control Algorithms

\begin{tabular}{|c|c|c|c|c|}
\hline $\begin{array}{l}\text { S. } \\
\text { No }\end{array}$ & Protocols & QoS & Limitations & $\begin{array}{l}\text { Simulation } \\
(\mathrm{Y} / \mathrm{N})\end{array}$ \\
\hline 1 & ACCP [14] & $\begin{array}{l}\text { Achieved } \\
\text { reliability } \\
\text { High throughput } \\
\text { Energy efficiency }\end{array}$ & $\begin{array}{l}\text { Limitations in } \\
\text { unilateral } \\
\text { approach }\end{array}$ & $\mathrm{Y}$ \\
\hline 2 & PCCP [33] & $\begin{array}{l}\text { Achieved high } \\
\text { link utilization } \\
\text { Better fairness } \\
\text { Decrease packet } \\
\text { loss } \\
\text { Improvement of } \\
\text { energy efficiency }\end{array}$ & $\begin{array}{l}\text { limitation in } \\
\text { managing } \\
\text { multiple sensed } \\
\text { data within a } \\
\text { node. } \\
\text { No mechanism in } \\
\text { heterogeneous } \\
\text { environment }\end{array}$ & $\mathrm{Y}$ \\
\hline 3 & $\begin{array}{l}\text { PHTCCP } \\
{[16]}\end{array}$ & $\begin{array}{l}\text { Desired } \\
\text { throughput } \\
\text { High link } \\
\text { utilization } \\
\text { Reduce packet } \\
\text { loss } \\
\text { Low packet drop } \\
\text { rate } \\
\text { Energy efficient }\end{array}$ & $\begin{array}{l}\text { Limit to maintain } \\
\text { reliability }\end{array}$ & $\mathrm{Y}$ \\
\hline 4 & $\begin{array}{l}\text { Reduced- } \\
\text { frame } \\
\text { TDMA } \\
\text { protocols [8] }\end{array}$ & $\begin{array}{l}\text { Improves the } \\
\text { maximum } \\
\text { throughput } \\
\text { Low level energy } \\
\text { consumption }\end{array}$ & $\begin{array}{l}\text { Limit to define } \\
\text { frame size }\end{array}$ & $\mathrm{Y}$ \\
\hline 5 & FACC [30] & $\begin{array}{l}\text { Improvement in } \\
\text { loss of packets, } \\
\text { Good energy } \\
\text { efficient, } \\
\text { Channel } \\
\text { utilization and } \\
\text { fairness }\end{array}$ & $\begin{array}{l}\text { Limitations in } \\
\text { handling } \\
\text { increased traffic } \\
\text { load / time } \\
\text { elapsed }\end{array}$ & $\mathrm{Y}$ \\
\hline 6 & $\begin{array}{l}\text { Hop-by-hop } \\
\text { traffic-aware } \\
\text { routing } \\
\text { scheme [4] }\end{array}$ & $\begin{array}{l}\text { Improvement in } \\
\text { end-to-end } \\
\text { packet delay, } \\
\text { network } \\
\text { performance, } \\
\text { packet delivery } \\
\text { ratio, and } \\
\text { energy }\end{array}$ & $\begin{array}{l}\text { Limitation of } \\
\text { traffic factors and } \\
\text { overhead }\end{array}$ & $\mathrm{Y}$ \\
\hline
\end{tabular}




\begin{tabular}{|c|c|c|c|c|}
\hline & & $\begin{array}{l}\text { consumption } \\
\text { Reduce } \\
\text { unnecessary } \\
\text { packet } \\
\text { retransmission } \\
\end{array}$ & & \\
\hline 7 & FDDC [6] & $\begin{array}{l}\text { Improve } \\
\text { performance in } \\
\text { data transfer, } \\
\text { Reduce data loss } \\
\text { and power saving } \\
\text { Increase network } \\
\text { performance } \\
\text { Speedup packet } \\
\text { transmission }\end{array}$ & $\begin{array}{l}\text { Limitation to } \\
\text { standardize } \\
\text { quantity of packet } \\
\text { Simulation not } \\
\text { performed }\end{array}$ & $\mathrm{N}$ \\
\hline 8 & $\begin{array}{l}\text { Multipath } \\
\text { Routing } \\
\text { Protocol [26] }\end{array}$ & $\begin{array}{l}\text { Prevents packet } \\
\text { loss } \\
\text { Smoothen traffic } \\
\text { Managed flow } \\
\text { control }\end{array}$ & $\begin{array}{l}\text { Limitations to } \\
\text { handle bursty } \\
\text { traffic }\end{array}$ & $\mathrm{N}$ \\
\hline 9 & RACC [35] & $\begin{array}{l}\text { Improve network } \\
\text { throughput } \\
\text { Fairness } \\
\text { performance }\end{array}$ & $\begin{array}{l}\text { Limitations of } \\
\text { window size } \\
\text { Time delay in } \\
\text { retransmission }\end{array}$ & $\mathrm{Y}$ \\
\hline 10 & ABS [17] & $\begin{array}{l}\text { Reduce packets } \\
\text { delay } \\
\text { Save energy due } \\
\text { to reduce packet } \\
\text { loss }\end{array}$ & $\begin{array}{l}\text { Limitations of } \\
\text { buffer occupancy } \\
\text { of n-hop } \\
\text { neighbours }\end{array}$ & $\mathrm{Y}$ \\
\hline
\end{tabular}

\section{Discussion}

The current discussion, describes the future directions which are important and required for the development of new mechanisms for congestion control in WSNs. The directive pursue the comparisons among the approaches as described in previous part and summarized in Table 1.

There are various solutions ranging from military to medical and for industry to home etc. WSN solutions categorized into event based, streaming based and query based. The event based applications (e.g. fire detection, target tracking wildlife monitoring etc.) are interactive, real-time, which generate the real time data from sensors which reaches to the data centre through sink node in short span of time for further proceedings. The multiple sensing nodes transmits the data, trafficking towards one sink, causes congestion around BS or sinks. So event based solutions can lead ephemeral congestion experience because of periodic and interim packet explode [9]. Many applications generates high volume of data. To handle such load, load balancing approaches is given to handle the network loads on established paths [24].

Similarly streaming based applications e.g. traffic monitoring, video surveillance, health monitoring etc. can be observed in multimedia WSNs [1]. The applications generate the high volume data, which require some certain bandwidth to tolerate at a certain extent otherwise it may cause congestion [2].

Query based applications push the data from supply node to base station by triggering an event. A query can also be used to administer the sensor nodes. It is understood that the instructions from sink comprise in one direction traffic and need high consistency. 
In WSN, concerning such applications, where a ephemeral congestion situation arises, congestion avoidance and mitigations mechanism must be accepted as a control methods for traffic. Various approaches has been employed [27, 7, 18, 33, 13]. On the other hand, if data load over traffic is distributed through alternative or multiple paths, then resource control methods are employed to control congestion [26, 28, 12].

We believe that a new congestion control algorithm need to develop which can consider the aforementioned directives as a base running applications over WSNs. It is also important to develop lightweight mechanism which should also be able to preserve the energy consumption in WSNs.

\section{Future Directions}

There are various critical tasks in which WSNs are expected to deploy to attain the real time objective. Due to unpredictable nature, an efficient congestion control method is required to support the applications of WSNs. By nature, the mechanism should be robust enough to adapt the scalability of WSN. Due to the memory and energy constrained nature of WSNs, it is expected to control the energy level and able to escalate the data from the sink to the data server without any end-to-end delay. Normally, it is observed that the packets are lost at sink level due to channel and buffer load. However various researchers have shown the routing, fairness and network utilization by simulation results but still Novel WSN congestion control mechanisms are expected to work on queue occupancy, buffer and channel load also. We believe the focus will be on suitable congestion control mechanisms, handling which can be achieved by development of performance controlled mechanisms in the vast area of WSNs.

\section{Conclusion}

Congestion control is one of the major concern area of research in WSNs. In this paper a critical analysis for the impact of congestion has been analyzed on various approaches (protocols, techniques, mechanisms) in WSNs along with the discussion and summarization of various attributes of protocols. By considering the demerits of existing challenges of approaches, some future directives is discussed and proposed for the performance improvisation of prospects mechanisms of congestion control.

\section{References}

[1] H. Shen and G. Bai, "Routing in wireless multimedia sensor networks: A survey and challenges ahead", Journal of Network and Computer Applications, doi: 10.1016/ j.jnca.2016.05.013, vol. 71, (2016), pp. 30-49.

[2] M. Hatamian, M. Asadboland, H. Barati, and M. A. Bardmily, "Congestion-Aware Routing and Fuzzybased Rate Controller for Wireless Sensor Networks”, Radio engineering, doi: 10.13164/re.2016-1, vol. 25, no. 1, (2016).

[3] S. A. Shah, B. Nazir and I. A. Khan, "Congestion control algorithms in wireless sensor networks: Trends and opportunities", Journal of King Saud University-Computer and Information Sciences, (2016).

[4] G. Majid, A. T. Haghighat and M. R. Meybodi, "Hop-by-hop traffic-aware routing to congestion control in wireless sensor networks", EURASIP Journal on Wireless Communications and Networking, doi 10.1186/s13638-015-0241-5, no. 1, (2015).

[5] M. A. Kafi, D. Djenouri, J. B. Othman and N. Badache, "Congestion control protocols in wireless sensor networks: a survey", IEEE Communications Surveys \& Tutorials, doi:10.1109/SURV.2014. 021714.00123, vol. 16, no. 3, (2014), pp. 1369-1390.

[6] M. Ashraf, S. Ameen and Prabakaran, "Flow Deviation Congestion Control in Wireless Sensor Networks", International Journal of Advanced Research in Computer Science and Software Engineering, vol. 4, no. 4, (2014), pp. 557-562.

[7] S. Guo, H. Zhang and Z. Zhong, "Detecting Faulty Nodes with Data Errors for Wireless Sensor Networks", ACM Transactions on Sensor Networks, doi: 10.1145/2594773, vol. 10, no. 3, (2014).

[8] D. J. Milica and G. L. Djordjevic, "Reduced-frame TDMA protocols for wireless sensor networks", International Journal of Communication Systems, vol. 27, no. 10, (2014), pp. 1857-1873. 
[9] C. Sergiou, P. Antoniou, and V. Vassiliou, "A Comprehensive Survey of Congestion Control Protocols in Wireless Sensor Networks", IEEE Communication Surveys \& Tutorials, vol. 16, no. 4, (2014), pp. 1839-1859.

[10] N. Thrimoorthy and T. Anuradha, "A Review on Congestion control Mechanisms in Wireless Sensor Networks", Int. Journal of Engineering Research and Applications, vol. 4, no. 2, (2014), pp. 54-59.

[11] S. Indurkar and N. P. Kulkarni, "Congestion Control in Wireless Sensor Networks: A survey", Int. Journal of Engineering Research and Applications, vol.4, no. 11, (2014), pp. 109-113.

[12] C. Sergiou and V. Vassiliou, "Estimating maximum traffic volume in wireless sensor networks using fluid dynamics principles", IEEE Commun. Lett., doi: 10.1109/ LCOMM.2013.010313.122281, vol. 17, no. 2, (2013), pp. 257-260.

[13] P. Antoniou, A. Pitsillides, T. Blackwell, A. Engelbrecht and L. Michael, "Congestion control in wireless sensor networks based on bird flocking behavior”, Comput. Netw. J., vol. 57, no. 5, (2013), pp. 1167-1191.

[14] G. J. Dzisi, D. K. Dake and K. Diawuo, “Adaptive congestion control protocol (ACCP) for wireless sensor networks", International Journal of Wireless \& Mobile Networks, vol. 5, no. 5, (2013), pp. 129144.

[15] A. Dâmaso, D. Freitas, N. Rosa, B. Silva and P. Maciel, "Evaluating the Power Consumption of Wireless Sensor Network Applications Using Models", Open Access Sensors, doi:10.3390/s130303473, vol. 13 no. 3, (2013), pp. 3473-3500.

[16] M. M. Mostafa, M. O. Rahman, A. S. K. Pathan and C. S. Hong, "Prioritized heterogeneous trafficoriented congestion control protocol for WSNs", Int. Arab J. Inf. Technol., vol. 9, no. 1, (2012), pp. 3948.

[17] D. S. Zaenab, K. Yoshigoe and R. B. Lenin, "Adaptive buffering scheme to reduce packet loss on densely connected WSN with mobile sink", Proceedings in IEEE Consumer Communications and Networking Conference (CCNC), Las Vegas, Nevada, USA, (2012).

[18] V. Zibakalam and M. H. Kahaei, "Increasing Throughput and Reducing Delay in Wireless Sensor Networks Using Interference Alignment", Int. J. Communications, Network and System Sciences, vol. 5, (2012), pp. 90-97.

[19] C. Cirstea, M. Cernaianu and A. Gontean, "Packet loss analysis in wireless sensor networks routing protocols", Proceedings of the IEEE 35th International conference on Telecommunication and Signal Processing (TSP), Evropska 15 Prague, Czech Republic, (2012).

[20] C. Sergiou, "Performance-aware congestion control in wireless sensor networks using resource control", Proceedings in IEEE 14th International Symposium and Workshops on World of Wireless, Mobile and Multimedia Networks (WoWMoM), Madrid, Spain, (2013).

[21] E. Dashkova and A. Gurtov, "Survey on congestion control mechanisms for wireless sensor networks", Proceedings in Internet of Things, Smart Spaces, and Next Generation Networking, Springer Berlin Heidelberg, (2012), pp. 75-85.

[22] P. Antoniou, "Nature-inspired congestion control and avoidance in wireless sensor networks", Ph.D. dissertation, University of Cyprus, Nicosia, Cyprus, (2012).

[23] A. M. Rooshenas, H. R. Rabiee, A. Movaghar and M. YousofNaderi, "Reducing The Data Transmission in Wireless Sensor Networks Using The Principal Component Analysis", Proceedings of the IEEE 6th International Conference on Intelligent Sensors, Sensor Networks and Information Processing (ISSNIP), Brisbane, Australia, (2010).

[24] M. Hao, T. Y. Ren-lai, L. S. Jiang and W. X. Dong, "Multipath routing protocol with load balancing in WSN considering interference", Proceedings of the 6th IEEE Conference on Industrial Electronics and Applications, Beijing, China, (2011).

[25] S. Sendra, J. Lloret, M. García and J. F. Toledo, "Power saving and energy optimization techniques for Wireless Sensor Networks", Journal of Communications, doi:10.4304/jcm. 6.6., vol. 6, no. 6, (2011), pp. 439-459.

[26] M. Cherian and T. R. G. Nair, "Multipath Routing with Novel Packet Scheduling Approach in Wireless Sensor Networks", IJCTE, doi: 10.7763/IJCTE.2011.V3.389, vol. 3, no. 5, (2011).

[27] W. W. Fang, J. M. Chen, L. Shu, T. S. Chu and D. P. Qian, "Congestion avoidance, detection and alleviation in wireless sensor networks", Journal of Zhejiang University SCIENCE C, doi:10.1631/jzus.C0910204, vol. 11, no. 1, (2010), pp. 63-73.

[28] K. Shi, Y. Shu, O. Yang and J. Luo, "Receiver-assisted congestion control to achieve high throughput in lossy wireless networks", IEEE Transactions on nuclear science, doi: 0.1109/TNS.2009.2037622, vol. 57, no. 2, (2010), pp. 491-496.

[29] M. Cherian and T. R. G. Nair, "A packet scheduling Strategy in Sensor Networks with SGMH Protocol", Proceedings of the IEEE International Conference on Computing Communication and Networking Technologies (ICCCNT), Karur, India, (2010).

[30] X. Yin, X. Zhou, R. Huang, Y. Fang and S. Li, "A fairness-aware congestion control scheme in wireless sensor networks", IEEE Transactions on Vehicular Technology, vol. 58, no. 9, (2009), pp. 5225-5234

[31] H. N. Dai, "Throughput and Delay in Wireless Sensor Networks using Directional Antennas", Proceedings of the IEEE International Conference on Intelligent Sensors, Sensor Networks and Information Processing (ISSNIP), Melbourne, Australia, (2009). 
[32] M. H. Yaghmaee and D. Adjeroh, "A reliable transport protocol for Wireless Sensor Networks", Proceedings of the IEEE International Symposium on Telecommunications, Tehran, Iran, (2008).

[33] W. Chongang, K. Sohraby, V. Lawrence, B. Li, and Y. Hu, "Priority-based congestion control in wireless sensor networks", Proceedings of the IEEE International Conference on Sensor Networks, Ubiquitous, and Trustworthy Computing (SUTC'06), Taichung, Taiwan, (2006).

[34] M. Zawodniok and S. Jagannathan, "Predictive congestion control MAC protocol for wireless sensor networks", Proceedings of the IEEE International Conference on Control and Automation, Budapest, Hungary, (2005).

[35] V. Bhuse, A. Gupta, and L. Lilien, "DPDSN: Detection of packet-dropping attacks for wireless sensor networks", Proceeding in Fourth Trusted Internet Workshop, Goa, India, (2005). 
International Journal of Multimedia and Ubiquitous Engineering Vol.12, No.1 (2017) 